\title{
GENETIC FACTORS ASSOCIATED WITH RISK AND DISABILITY PROGRESSION OF MULTIPLE SCLEROSIS IN SLOVAK POPULATION
}

\author{
HaNYSOVA $\mathrm{S}^{1}$, CIERNY D ${ }^{2}$, KuRCA E $\mathrm{E}^{3}$, LEHOTSKY J., \\ ${ }^{1}$ Department of Medical Biochemistry, Jessenius Faculty of Medicine in Martin, Comenius University in Bratislava, \\ Slovakia \\ ${ }^{2}$ Department of Clinical Biochemistry, Jessenius Faculty of Medicine in Martin, Comenius University in Bratislava \\ and University Hospital Martin, Slovakia \\ ${ }^{3}$ Department of Neurology, Jessenius Faculty of Medicine in Martin, Comenius University in Bratislava and \\ University Hospital Martin, Slovakia \\ ${ }^{4}$ Biomedical Center Martin, Jessenius Faculty of Medicine in Martin, Comenius University in Bratislava, Slovakia
}

\begin{abstract}
Objective: The aim of our study was to determine the relation of particular genetic variants in selected genes (GSTM1, GSTT1 null genotypes; rs 1695 GSTP1; rs 10735781 EVI5) to the risk of multiple sclerosis (MS) development and find out the possible association with disease disability progression rate.

Material and methods: Our study included 202 MS patients and 174 healthy control volunteers. MS patients were divided according to disability progression rate to three groups - slowly progressing, mid-rate progressing and rapidly progressing. All DNA samples were isolated from venous blood. Genotyping was performed by PCR-RFLP and multiplex PCR.

Results: Our analysis showed that GSTT1 null genotype (OR 0.56; 95\%CI $0.33-0.95 ; \mathrm{p}=0.04$ ) and GSTM1, GSTT1 double null genotype (OR 0.32; $95 \% \mathrm{CI} 0.14-0.74 ; \mathrm{p}=0.006)$ are potentially protective in relation to MS. We observed similar result in GSTT1 null genotype in association with mid-rate progression (OR 0.48; 95\%CI $0.24-0.97 ; \mathrm{p}=0.05$ ). Frequency of GSTM1 and GSTT1 double null genotype is significantly lower in subgroup of MS patients with progression rate defined as slow (OR 0.22; 95\%CI $0.05-0.98 ; \mathrm{p}=0.05$ ) and middle (OR 0.33; 95\%CI $0.11-0.99 ; \mathrm{p}=0.045$ ). We did not show any significant association of genetic changes rs 1695 in GSTP1 and rs 10735781 in EVI5 with MS or rate of disease progression.

Conclusions: Genetic basis of multiple sclerosis is still not fully elucidated. Further research may clarify our results and confirm the value of studied factors for clinical practice.
\end{abstract}

Key words: multiple sclerosis, MSSS score, null genotype, gene polymorphism, PCR analysis

\section{INTRODUCTION}

Multiple sclerosis (MS) is a progressive autoimmune disease of central nervous system characterized by neuroinflammation, demyelination and neurodegeneration. Factors triggering the disease onset and modulating variable disability progression rate in individual patients are still not fully elucidated. The MS symptoms are heterogenous and ambiguous, associated with the degree and localization of nerve tissue damage. Generally, there are distinguished several clinical MS forms, with the highest prevalence of relapsing-remitting form.

It is proposed that for the MS etiopathogenesis are responsible immune, environmental and genetic factors. Genetic factors associated with MS can be generally classified into HLA (human leukocyte antigen) genes and non-HLA genes [1]. Genetic predisposition to MS is represented mainly by a allele HLA-DRB1 * 1501 , which is widely accepted as the most important susceptibility allele of MS [2,3]. Gene products of non-HLA genes can contribute to the genetic risk of

Address for correspondence:

RNDr. Sandra Hanysova; Department of Medical Biochemistry, Jessenius Faculty of Medicine in Martin, Comenius University in Bratislava, Mala Hora 4, 03601 Martin, Slovakia;phone+42 1-43-2633-659;

e-mail: hanysova@jfmed.uniba.sk; sandrahanysova@zoho.com 
MS by modulation of different processes, including the regulation of $\mathrm{T}$ and $\mathrm{B}$ cells functions, cytokine signalization, oxidative stress, metabolism of vitamin D, iron metabolism, neuronal regeneration and many others [1].

The aim of the present study was the analysis of selected non-HLA genetic variations in Slovak relapsing-remitting MS patients. Our choice included three antioxidant enzymes, known as glutathione-S-transferases (rs1695 GSTP1, null genotypes of GSTM1, GSTT1) and one candidate marker, single nucleotide polymorphism rs10735781 of EVI5 gene. EVI5 (ecotropic viral integration site 5) is a protein-coding gene, EVI5 protein is important for mitotic cytokinesis and cell signalization in immune pathways [4,5]. All mentioned genetic changes have the potential association with disease onset or progression rate.

\section{METHODS}

Our study included 202 MS patients (age $39.86 \pm 8.85$ ) and 174 healthy control volunteers (age $34.94 \pm 10.18$ ). To evaluate the disease progression rate in patients, we used the widely accepted Multiple Sclerosis Severity Score (MSSS, range from 0.01 to 9.99) [6] that considers the neurological impairment of the functional systems (Expanded Disability Status Scale, EDSS) [7] together with disease duration. For the purposes of the association of these markers with the rate of disease disability progression, we stratified MS patients by MSSS score to three groups - slowly progressing MS (MSSS $<3 ; n=71$ ), mid-rate progressing MS (MSSS 3-6; $\mathrm{n}=98$ ) and rapidly progressing MS (MSSS $>6 ; \mathrm{n}=33$ ). DNA from each patient was isolated from whole blood by commercial kit. Genotyping was performed by multiplex PCR reaction or PCR-RFLP method, with subsequent separation of amplified DNA fragments by gel electrophoresis. Primer sequences for multiplex PCR reaction: GSTM1 forward $5^{\prime}-$ GAACTCCCTGAAAAGCTAAAGC-3' and reverse 5'-GTTGGGCTCAAATATACGGTGG-3'; GSTT1 forward 5' $5^{\prime}$-TTCCTTACTGGTCCTCACATCTC-3' and reverse 5' ${ }^{\prime}$-TCACCGGATCAT GCCAGCA-3'; control fragment forward 5'-CAACTTCATCCACGTTCACC-3' and reverse 5'-GAAGAGCCAAGGACAGGTAC-3'. rs 1695 and rs10735781 were detected by PCR-RFLP method. Primer sequences for rs 1695 GSTP1: forward 5'-GTAGTTTGCCCAAGGTCAAG-3' and reverse $5^{\prime}$-AGCCACCTGAGGGGTAAG-3', restriction cleavage was done by Alw26I enzyme. Primer sequences for rs 10735781 EVI5: forward 5'-CCCCTCAGACTAACAGTGGA-3' and reverse 5' -TTTGGTTTCTGTTGGTGTGG-3', restriction cleavage was proceeded by AluI enzyme. Statistical analysis of the studied data was done using contingency tables with estimation of odds ratio and 95\% confidence interval for the degree of association between two variables, $p$ value was calculated by Fisher exact test and level of statistical significance was assumed as $\mathrm{p} \leq 0.05$.

\section{RESULTS}

Our analysis of GSTM1 and GSTT1 null genotypes individually or in combination showed significant result in lower GSTT1 null genotype frequency in MS patients (13.9\% vs. $22.4 \%$ in controls; OR 0.56; 95\%CI $0.33-0.95 ; \mathrm{p}=0.04)$. Significant result was also detected in GSTM1, GSTT1 double null genotype (4\% in MS patients vs. $11.5 \%$ in controls; OR 0.32; $95 \%$ CI $0.14-0.74 ; \mathrm{p}=0.006$ ). Our results indicate that GSTT1 null genotype and double null genotype are protective in relation to multiple sclerosis. Focusing on various disease disability rate, GSTM1 null genotype is not predictive for any progression rate ( $>0.05)$ and GSTT1 null genotype is predictive in association with middle progression rate (OR $0.48 ; 95 \%$ CI 0.24 - 0.97; $p=0.05$ ), another results were not identified as significant ( $p>0.05$ ). Frequency of GSTM1, GSTT1 double null genotype is significantly lower in subgroup of MS patients with slow progression rate (OR 0.22; 95\%CI $0.05-0.98 ; \mathrm{p}=0.05$ ) and middle (OR 0.33; 95\%CI $0.11-0.99 ; \mathrm{p}=0.045)$. 
In rs 1695 GSTP1, we did not show any significant association of risk recessive allele G $(27.7 \%$ in MS patients vs. $28.4 \%$ in controls; OR 0.96; $95 \%$ CI $0.70-1.33 ; \mathrm{p}=0.87$ ) or genotype GG (3\% in MS patients vs. 7\% in controls; OR 0.41; $95 \%$ CI $0.15-1.13$; $p=0.09$ ) with multiple sclerosis. Genotype analysis focusing on potential association of recessive allele or genotype frequencies with disease disability rate stratified by MSSS score did not indicate significant results (p>0.05). Studying of rs 10735781 EVI5 also did not suggest any association of this polymorphism with the multiple sclerosis (risk allele G 44\% in MS patients vs. $42 \%$ in controls; OR 1.07; $95 \%$ CI 0.8 - 1.42; p=0.71; risk genotype GG 16.8\% in MS patients vs. $12.6 \%$ in controls; OR 1.40; $95 \%$ CI $0.78-2.45 ; \mathrm{p}=0.31)$ or progression rate $(\mathrm{p}>0.05)$.

\section{DISCUSSION}

The study of new MS genetic markers with possible diagnostic or prognostic importance, which may be helpful for early detection and monitoring of disease course or therapeutic responses, is still needed. Despite the huge research efforts, genetic basis of multiple sclerosis, which is considered to be the multifactorial disease, is still not clear, especially in cases of non-HLA candidate mutations or polymorphisms. Concerning to the Slovak MS patients, there are some results about non-HLA genetic changes. Allele C of rs6897932 in interleukin 7 receptor alpha chain (IL7Ra) gene was present in the higher frequency in MS patients (OR 1.31; $95 \%$ CI $1.0-1.72 ; \mathrm{p}=0.047$ ) and allele $\mathrm{T}$ is considered to be protective against MS development (OR 0.76; 95 \% CI 0.58 - 0.10; p=0.047). The genotype analysis showed that MS patients manifested a lower frequency of genotype CT when compared to controls or genotype TT and there was also been detected higher frequency of genotype CC. Authors found a significantly decreased risk of MS development in carriers of allele $\mathrm{T}$ with genotype CT (OR 0.87, 95 \% CI 0.61 - 1.23; p=0.05) or TT (OR 0.565; 95 \% CI 0.28 - 1.13; $\mathrm{p}=0.05$ ). Moreover, minor allele T and genotype TT are protective against rapid disease disability progression in MS [8]. The findings about rs 10735810 in vitamin D receptor gene in Slovak MS patients have shown the association of heterozygous FokI Ff genotype with increased risk of MS in women (OR 1.48; $95 \%$ CI $1.01-2.16$; $\mathrm{p}=0.042$ ). Despite it, there have not been found statistically significant differences in the proportions of FokI genotypes or allele frequencies between total MS patient and the control group. Authors also observed a trend of higher frequency of homozygotes FF in MS patients with rapid disease progression in comparison to the slow progressing MS patients (OR 1.93; $95 \%$ CI $0.94-$ $3.94 ; \mathrm{p}=0.071$ ) [9]. The aim of our work was to analyse selected genetic changes (GSTM1, GSTT1 null genotypes; rs 1695 GSTP1, rs 10735781 EVI5) in a group of Slovak MS patients and to shed light on their potential importance for disease onset or disability rate. Certain genetic variations of glutathione-S-transferases with the negative influence on enzyme functions have been previously indicated as associated with multiple sclerosis, but their role in the etiopathogenesis of MS still remains controversial [10-12]. In group of 455 Serbian MS patients, GSTT1 null genotype and GSTM1, GSTT1 double null genotypes were observed in significantly higher frequency ( $\mathrm{p}<0.0001$ and $\mathrm{p}<0.05)$ when compared to 366 healthy people [12]. Another results from study of patients with North-European [11] or Greek origin [13] did not identify any relevant association of these changes with multiple sclerosis. Our results indicate the importance of GSTT1 null genotype $(\mathrm{p}=0.04)$ and double null genotype GSTM1, GSTT1 (p=0.006) for MS. Higher prevalence of null genotypes in control group suggests the protective role of these changes for MS onset and development, which is not in line with previously mentioned data [12]. We obtained significant result in GSTT1 null genotype and its association with mid-rate progression $(\mathrm{p}=0.05)$, also the frequency of GSTM1, GSTT1 double null genotype is significantly lower in subgroup of MS patients with progression rate defined as slow $(\mathrm{p}=0.05)$ and middle $(\mathrm{p}=0.045)$. GSTM1 null genotype did not show any significant association with disease onset or progression rate (p>0.05). 
rs 1695 of GSTP1 gene is a missense mutation with considerably negative impact on the enzyme activity and decreasing the catalytic functions. Research group [10] showed the significant association ( $\mathrm{p}<0.05)$ of double recessive genotype ( $\mathrm{rs} 1695$ and $\mathrm{rs} 1800566$ in NADPH quinine oxidoreductase 1) with the insufficient therapeutical response in group of 130 Greek MS patients. In group of North-European multiple sclerosis patients with disease duration more than 10 years has been observed significantly higher frequency of rs 1695 dominant AA genotype and GSTM1 null genotype in combination ( $p=0.022)$ [11]. Another study focusing on rs 1695 in Croatian MS patients did not prove any significant relation of this mutation with multiple sclerosis [14]. Based on our results, we did not suppose any significant association of recessive allele or genotype with MS development or disability progression in group of Slovakian patients ( $\mathrm{p}>0.05)$.

According to ambiguity of available data $[11,13,14]$ and highly polymorphous occurence of various changes in GST gene sequences in healthy population, which is accepted in general, we did not suggest direct association of these gene alterations with the MS onset or progression. Further research is needed for supporting these answers.

rs 10735781 of EVI5 gene is the polymorphism indicated as predictive marker for multiple sclerosis. The association of risk genotype GG with European MS patients has been proved by association studies and meta-analyses [15-17]. Significant results are described in Dutch population ( $\mathrm{p}=0.02)$ [16] and also by genome-wide association study of MS patients with English or American origin ( $p=0.000335)$ [15]. Similar results about the association of risk genotype GG with disease onset are showed in Americans with African origin ( $p=0.006)$ [17]. Results of our work are not in line with previously mentioned data. We did not find any significant association of risk allele $\mathrm{G}$ or genotype GG with disease onset or disability progression in Slovak MS patients ( $\mathrm{p}>0.05)$.

In summary, we can conclude that particular non-HLA genetic variants can be important for evaluation of the risk of MS development and disability progression. Positive or negative prognostic genetic markers also improve the diagnostic and therapeutic procedure and can help to minimize neurological damage in predisposed individuals.

\section{REFERENCES}

1. Nischwitz S, Müller-Myhsok B, Weber F. Risk conferring genes in multiple sclerosis. FEBS Lett. 2011; 585 (23): 3789-97.

2. Sawcer S, Hellenthal G, Pirinen M, Spencer CCA, Patsopoulos NA, Moutsianas L, et al. The International Multiple Sclerosis Genetics Consortium (IMSGC), Wellcome Trust Case Control Consortium 2 (WTCCC2). Genetic risk and a primary role for cell-mediated immune mechanisms in multiple sclerosis. Nature. 2011; 476 (7359): 214-9.

3. Zhang Q, Lin CY, Dong Q, Wang J, Wang W. Relationship between HLA-DRB1 polymorphism and susceptibility or resistance to multiple sclerosis in Caucasians: a meta-analysis of non-family-based studies. Autoimmun Rev. 2011; 10 (8): 474-81.

4. Faitar SL, Sossey-Alaoui K, Ranalli TA, Cowell JK. EVI5 protein associates with the INCENP-aurora B kinase-survivin chromosomal passenger complex and is involved in the completion of cytokinesis. Exp Cell Res. 2006;312 (12): 2325-35.

5. Mowry EM, Carey RF, Blasco MR, Pelletier J, Duquette P, Villoslada P, et al. Multiple sclerosis susceptibility genes: associations with relapse severity and recovery. PLoS One. 2013; 8 (10): e75416.

6. Roxburgh RH, Seaman SR, Masterman T, Hensiek AE, Sawcer SJ, Vukusic S et al. Multiple sclerosis severity score: using disability and disease duration to rate disease severity. Neurology. 2005; 64 (7): 1144-51.

7. Kurtzke JF. Rating neurologic impairment in multiple sclerosis: an expanded disability status scale (EDSS). Neurology. 1983; 33 (11):1444-52.

8. Čierny D, Hányšová S, Michalik J, Kantorová E, Kurča E, Škereňová M, Lehotský J. Genetic variants in interleukin 7 receptor chain (IL-7Ra) are associated with multiple sclerosis risk and disability progression in Central European Slovak population. J Neuroimmunol. 2015; 282: 80-4. 
9. Čierny D, Michalik J, Kurča E, Dobrota D, Lehotský J. FokI vitamin D receptor gene polymorphism in association with multiple sclerosis risk and disability progression in Slovaks. Neurol Res. 2015; 37 (4): 301-8.

10. Alexoudi A, Zachaki S, Stavropoulou C, Gavrili S, Spiliopoulou C, Papadodima S et al. Possible Implication of GSTP1 and NQO1 Polymorphisms on Natalizumab Response in Multiple Sclerosis. Ann Clin Lab Sci. 2016; 46 (6): 586-591.

11. Mann CL, Davies MB, Boggild MD, Alldersea J, Fryer AA, Jones PW et al. Glutathione S-transferase polymorphisms in MS: their relationship to disability. Neurology. 2000; 54(3): 552-7.

12. Živković M, Životić I, Dinčić E, Stojković L, Vojinović S, Stanković A. The glutathione S-transferase T1 deletion is associated with susceptibility to multiple sclerosis. J Neurol Sci. 2013; 334(1-2):6-9.

13. Stavropoulou C, Korakaki D, Rigana H, Voutsinas G, Polyzoi M, Georgakakos VN et al. Glutathione-Stransferase T1 and M1 gene polymorphisms in Greek patients with multiple sclerosis: a pilot study. Eur J Neurol. 2007; 14(5): 572-4.

14. Bačić Baronica K, Mlinac K, Petlevski R, Ozretić D, Vladić A, Kalanj-Bognar S, Zuntar I. Progression of multiple sclerosis is associated with gender differences in glutathione S-transferase P1 detoxification pathway. Acta Neurobiol Exp (Wars). 2014; 74(3): 257-65.

15. Hafler DA et al., INTERNATIONAL MULTIPLE SCLEROSIS GENETICS CONSORTIUM 1. Risk alleles for multiple sclerosis identified by a genomewide study. N Engl J Med. 2007; 2007357 (9): 851-62.

16. Hoppenbrouwers IA, Aulchenko YS, Ebers GC, Ramagopalan SV, Oostra BA, Van Duijn CM, Hintzen RQ. EVI5 is a risk gene for multiple sclerosis. Genes Immun. 2008; 9 (4): 334-7.

17. Johnson BA, Wang J, Taylor EM, Caillier SJ, Herbert J, Khan OA et al. Multiple sclerosis susceptibility alleles in African Americans. Genes Immun. 2010; 11 (4): 343-50.

Acknowledgement: This work was supported by grants APVV 15-0107 and ITMS: 26220220114 Identification of novel markers in diagnostic panel of neurological diseases.

Received: July, 21, 2017

Accepted: August, 31, 2017 